\title{
Event-driven and Attribute-driven Robustness
}

\begin{abstract}
M. Namakshenas ${ }^{1}$, M.S. Pishvaee,,, M. Mahdavi Mazdeh ${ }^{3}$
Over five decades have passed since the first wave of robust optimization studies conducted by Soyster and Falk. It is outstanding that real-life applications of robust optimization are still swept aside; there is much more potential for investigating the exact nature of uncertainties to obtain intelligent robust models. For this purpose, in this study, we investigate a more refined description of the uncertain events including (1) event-driven and (2) attribute-driven. Classical methods transform convex programming classes of uncertainty sets. The structural properties of uncertain events are analyzed to obtain a more refined description of the uncertainty polytopes. Hence, tractable robust models with a decent degree of conservatism are introduced to avoid the over-protection induced by classical uncertainty sets.
\end{abstract}

Keywords: Robust optimization, Convex optimization, Uncertainty sets, Uncertainty events.

Manuscript was received on 01/06/2017 revised on 27/08/2017 and accepted for publication on 09/09/2017

\section{Introduction}

Robust optimization is a tractable alternative to stochastic programming, particularly suited for the problems in which parameters are unknown and their respective distributions are uncertain. In many real-world situations, a precise stochastic description of the uncertain events may not be available. With less structured information, such as bounds of uncertain parameters, one might describe the existing uncertainties by dedicating a set in which all realizations should lie, i.e., "uncertainty set". The goal is to guarantee the feasibility of the underlying constraints for any possible realization while optimizing an objective defending against the worst possible consequence.

The original form of robust optimization, introduced by Soyster [10] and Falk [6], was generally concerned with linear programming problems with inexact technological coefficients. Their proposed robust optimization was too conservative and subjected to being drawn to the worst case for each uncertain parameter since the considered uncertainty was limited to a column-wise structure. Numerous works significantly generalized and extended the earlier platform into other classes of convex optimization problems beyond linear programming; e.g., conic and semi-definite programming (for example, see [2] and [5]). The other works paved the way for a more complex description of the uncertainty polytopes; e.g., intersections of ellipsoidal uncertainty sets, budgeted uncertainty sets, etc. (for example, see [2] and [4]). The key idea behind an uncertainty set is based on three components: nominal values of uncertain parameters, perturbation values, and uncertainty generating mechanism.

\footnotetext{
* Corresponding Author.

1 School of Industrial Engineering, Iran University of Science and Technology, Tehran, Iran, E-mail: m_namakshenas@ind.iust.ac.ir.

2 School of Industrial Engineering, Iran University of Science and Technology, Tehran, Iran, E-mail: pishvaee@iust.ac.ir.

3 School of Industrial Engineering, Iran University of Science and Technology, Tehran, Iran, E-mail: mazdeh@iust.ac.ir.
} 
Our work is mainly focused on the uncertainty set proposed by Bertsimas and Sim [5], the socalled "cardinality-restricted uncertainty set":

$$
S=\left\{e \in R^{n}: e_{i}=\bar{e}_{i}+\delta_{i} \hat{e}_{i}, 0 \leq \delta_{i} \leq 1, \sum_{i} \delta_{i} \leq \Gamma\right\},
$$

where the $\overline{e_{i}}$ are the nominal values, the $\hat{e}_{i}$ are the perturbed values of uncertain parameters, $\delta_{i}$ are the cardinality controlling variables, and $\Gamma$ is the budget of uncertainty. Our goal is to investigate more refined versions of uncertainty sets. Hence, it enables us to avoid the over-protection issued by the classic cardinality-restricted uncertainty set. The generating polytope of the classic version benefits from convexity, especially integrality of its convex hull, which makes it more tractable. However, it is independent of decision variables of the robust model and the type of the uncertainty event. In this study, we present less conservative uncertainty sets which improve the protection level of the classic version; i.e., set $S$. We discuss the types of events which generate the combinatorial structure of uncertainty sets; i.e., "combinatorial uncertainty set". We also address the tractability issue of some problems caused by proposed uncertainty sets. A strategy is proposed to convert the event-driven uncertainty polytopes into attribute-driven ones, which facilitates the transformation of the original model into a tractable robust counterpart.

The rest of our work is organized as follows. Section 2 investigates the structural properties generated by the two common types of uncertainty sets. Section 3 applies the discussed uncertainty sets to two classic problems: robust knapsack problem and robust portfolio selection problem. The last section concludes the study with a summary and future directions.

\section{Structural Properties of Uncertain Events}

In this section, we briefly discuss the structural properties generated by the two common types of uncertainty sets, naming them "attribute-driven uncertainty set" (data-driven uncertainty set) and "event-driven uncertainty set" (combinatorial uncertainty set).

Attribute-driven uncertainty sets use the perturbed values of uncertain parameters as direct inputs to the mathematical model of the robust counterpart. They connect the decision maker's risk preferences with the "budget of uncertainty" and the controlling mechanism of uncertainty. For example, consider the portfolio selection problem in which an investor chooses the proportion of capital to be invested in each of $N$ assets such that a desired wealth is achieved. The objective is to determine the fraction of invested asset $i$ so as to maximize the total portfolio return. An underlying assumption of Markowitz's model is that precise estimates of return of asset $i, \mu_{i}$, and risk of asset $i$, $\sigma_{i}$, have been obtained. However, asset returns are uncertain. Hence, we can interpret risk of return $i$ as the perturbed value of return of asset $i$ and incorporate it in the uncertainty set,

$$
S=\left\{\tilde{\mu} \in R^{n}: \tilde{\mu}_{i}=\bar{\mu}_{i} \pm \delta_{i} \sigma_{i}, 0 \leq \delta_{i} \leq 1, \sum_{i} \delta_{i} \leq \Gamma\right\} .
$$

In this example, the risks act as "internal" functions of asset returns, and any value of the variable $\delta_{i}$ enforces asset $i$ to gain the risk value $i$. 
On the other hand, the event-driven uncertainty set triggers uncertainty in parameters when a specific event occurs in the system and generates discrete uncertainties,

$$
U=\left\{\delta \in\{0,1\}^{|J|}, \sum_{j \in J} \delta_{j} \leq \Gamma\right\}
$$

The uncertainty set $U$ is non-convex and the variable $\delta$ plays a key role in generating the combination of events. The given set $J$ includes entities generating independently dichotomous events; e.g., happened and not happened, and parameter $\Gamma \in \mathbb{Z}$ is the maximum number of these events. The disruption systems are great examples of this type of uncertainty sets. An et al. [1] used a combinatorial uncertainty set to formulate a $p$-median facility location problem prone to disruptions. Moreover, the uncertainty set $U$ can be modified in order to encompass the data of uncertain parameters. However, incorporating an event-driven uncertainty set into a mathematical model could make it computationally intractable. There exist two types of strategies, to the best of our knowledge, to solve the transformed model. One approach is mainly based on iterative algorithms. Zeng [12] proposed a column-and-constraint generation algorithm to solve the twostage robust optimization problems in which the first stages defined by a combinatorial uncertainty set. They also solved the model with existing benders-style cutting plane methods.

The second type of methods, as originally presented here, transform event-driven uncertainty polytopes into attribute-driven ones. They are heuristically performed and differ from one problem to another. The proposed strategy paves the way for transformation of the original model into a tractable robust counterpart. We discuss this method in the next section.

\section{Event and Attribute-driven Robustness}

\subsection{Robust Event-driven Knapsack Problem}

Consider a knapsack problem, with given parameters $c_{i}$ as the costs and $w_{i}$ as the weight of item $i$ :

$$
\begin{aligned}
\text { Maximize } & \sum_{i \in N} c_{i} x_{i} \\
\text { s.t. } & \sum_{i \in N} \tilde{w}_{i} x_{i} \leq b \\
& x_{i} \in \mathrm{Z}^{+}, \quad \forall i \in N .
\end{aligned}
$$

It is assumed that the weights $w_{i}$ are uncertain; identically, independently, and uniformly distributed in $\left[\bar{w}_{i}, \bar{w}_{i}+\hat{w}_{i}\right]$. The objective value coefficient vector $c$ is not subject to data uncertainty. The goal is to maximize the total utility of $|N|$ items. The item should be selected and loaded on a cargo with strict weight restrictions. Suppose item $i$ with weight $\bar{w}_{i}$ has auxiliary component $i$ with weight $\hat{w}_{i}$. If uncertainty event occurs, $\delta_{i}=1$, the weight of item $i$ should be increased by $\hat{w}_{i}$. We define the uncertainty set as follows.

$$
U^{\Gamma}=\left\{w \in R^{|N|}: \tilde{w}_{i}=\bar{w}_{i}+\delta_{i} \hat{w}_{i}, \delta_{i} \in\{0,1\}, \sum_{i} \delta_{i} \leq \Gamma\right\} .
$$


Then, the original robust problem with respect to the worst-case criterion becomes

$$
\begin{aligned}
\text { Maximize } & \sum_{i \in N} c_{i} x_{i} \\
\text { s.t. } & \sum_{i \in N} \bar{w}_{i} x_{i}+\max _{U^{\Gamma}}\left\{\sum_{j \in N} \hat{w}_{j} x_{j}^{*}\right\} \leq b \\
& x_{i} \in \mathrm{Z}^{+}, \quad \forall i \in N .
\end{aligned}
$$

In order to solve the robust part of the problem (inner maximization), we heuristically define a second problem that seeks the worst-case outcome when the combination of events occurs:

$$
\begin{aligned}
\text { Maximize } & \sum_{j \in N} \hat{w}_{j} \delta_{j} x_{j}^{*} \\
\text { s.t. } & \sum_{j \in N} \delta_{j} \leq \Gamma \\
& \delta_{j} \in\{0,1\}, \quad \forall j \in N .
\end{aligned}
$$

In order to reach a closed form of the heuristic problem (7), we need to take the dual form of it. At the first glance, the dualization technique may not be applicable since the problem is not a convex linear programming one (variable $\delta_{i}$ is defined over integer domain). The following property addresses the integrality issue caused by the integer variables (we name this problem as “ $\Xi ")$.

Property 3.1. The convex hull of $\Xi$ is an integral polyhedron.

Proof. See the Appendix.

Now, we extract the dual form of relaxed $\Xi$ and incorporate it into the original problem. Relaxed $\Xi$ is feasible and bounded for all $\Gamma \in[0,|N|]$. Also, the dual of the relaxed $\Xi$ is feasible and bounded (and by strong duality, the objective values coincide):

$$
\begin{aligned}
\text { Maximize } & \sum_{i \in N} c_{i} x_{i} \\
\text { s.t. } & \sum_{i \in N} \bar{w}_{i} x_{i}+\tau \Gamma+\sum_{i} p_{i} \leq b \\
& p_{i}+\tau \geq \hat{w}_{i} x_{i}, \quad \forall i \in N \\
& \tau, p_{i} \geq 0, \quad \forall i \in N \\
& x_{i} \in \mathrm{Z}^{+}, \quad \forall i \in N,
\end{aligned}
$$

where $\tau$ and $p_{i}$ are the dual variables of the relaxed $\Xi$.

\subsection{Variable Budgeted Uncertainty Set}

In practical cases, a limited "budget pool" is dedicated to both the item selection process and the robustness cost. In the previous example, the user only defines the budget of robustness, whereas 
the whole budget must be simultaneously addressed. Consider the previous example, the cargo (knapsack) problem. Suppose the cargo carries two set of items: humanitarian items, П, and commercial products, Í. One cannot consider the underlying uncertainty of commercial products when necessary products are not loaded. On the other hand, to avoid the over-protection caused by $U^{\Gamma}$, which is independent of $x$, Poss [9] introduced a novel model of uncertainty polytope. Instead of considering a fixed budget of uncertainty $\Gamma$, a multifunction of $x, \gamma(x)$, was considered. We made some modifications to incorporate the variable budget into our uncertainty set. The objective still seeks to maximize the total utility of items, given $\Phi$ as the total budget for the robustness,

$$
\Phi=\alpha \sum_{i \in \Pi} \operatorname{sign}\left(x_{i}^{*}\right)
$$

where $\alpha$ is the robustness importance. The budget of uncertainty varies depending on the selected items, $\operatorname{sign}\left(x_{i}^{*}\right)$, from the set $\Pi$. Then, the variable budgeted uncertainty set with the event-driven approach is proposed as follows:

$$
U^{\Phi}=\left\{\tilde{w} \in R^{|N|}: \tilde{w}_{i}=\bar{w}_{i}+\delta_{i} \hat{w}_{i}, \delta_{i} \in\{0,1\}, \sum_{i \in N} \delta_{i} \leq \Phi\right\} .
$$

According to Property 3.1 and the worst-case criterion, the robust counterpart of this problem can be formulated as follows.

$$
\begin{aligned}
\text { Maximize } & \sum_{i \in N} c_{i} x_{i} \\
\text { s.t. } & \sum_{i \in N} \bar{w}_{i} x_{i}+\alpha \tau \sum_{i \in \Pi} \operatorname{sign}\left(x_{i}\right)+\sum_{i \in N} p_{i} \leq b \\
& p_{i}+\tau \geq \hat{w}_{i} x_{i}, \quad \forall i \in N \\
& \tau, p_{i} \geq 0, \quad \forall i \in N \\
& x_{i} \in \mathrm{Z}^{+}, \quad \forall i \in N
\end{aligned}
$$

where $\tau$ and the $p_{i}$ are the dual variables. Note that the first constraint contains a nonlinear expression. We make the following modifications and insertions in order to have a tractable linear form of the proposed counterpart:

$$
\begin{gathered}
y_{i}=\operatorname{sign}\left(x_{i}\right), \quad \forall i \in \Pi \\
x_{i} \leq\left[\frac{b}{\bar{w}_{i}}\right] y_{i} ; \quad x_{i} \geq y_{i}, \quad \forall i \in \Pi \\
\sum_{i \in \Pi} \lambda_{i}=\tau \sum_{i \in \Pi} y_{i}, \\
\lambda_{i} \geq-\mathbf{M}\left(1-y_{i}\right)+\tau, \quad \forall i \in \Pi \\
\lambda_{i} \geq 0, \quad y_{i} \in\{0,1\}, \quad \forall i \in \Pi .
\end{gathered}
$$

The linear robust counterpart is presented as follows: 


$$
\begin{aligned}
\text { Maximize } & \sum_{i \in N} c_{i} x_{i} \\
\text { s.t. } & \sum_{i \in N} w_{i} x_{i}+\alpha \sum_{\forall i \in \Pi} \lambda_{i}+\sum_{i \in N} p_{i} \leq b \\
& p_{i}+\tau \geq \hat{w}_{i} x_{i}, \quad \forall i \in N \\
& x_{i} \leq\left[\frac{b}{\bar{w}_{i}}\right] y_{i}, \quad \forall i \in \Pi \\
& x_{i} \geq y_{i}, \quad \forall i \in \Pi \\
& \lambda_{i} \geq-\mathbf{M}\left(1-y_{i}\right)+\tau, \quad \forall i \in \Pi \\
& \tau, p_{i} \geq 0, \quad \forall i \in N \\
& x_{i} \in \mathrm{Z}^{+}, \quad \forall i \in N \\
& \lambda_{i} \geq 0, \quad \forall i \in \Pi \\
& y_{i} \in\{0,1\}, \quad \forall i \in \Pi
\end{aligned}
$$

Property 3.2. The constraints set (15) do not impose any additional restriction on the minimal value of $\tau$ so that we can choose $\mathbf{M}$ equal to $\max _{i \in N}\left(\hat{w}_{i}\right)$, and write

$$
\lambda_{i} \geq-\max _{i \in N}\left(\hat{w}_{i}\right)\left(1-y_{i}\right)+\tau, \quad \forall i \in \Pi
$$

Property 3.3. Model (11) can be easily verified with setting $\alpha=1$ and $|\Pi|=|N|=\Gamma$. It is immediate that $\operatorname{opt}\left(U^{\Gamma}\right)=\operatorname{opt}\left(U^{\Phi}\right)$.

\subsection{Risk Compensatory Uncertainty Set}

Bertsimas and Sim [4] reformulated a maximum expected return of a portfolio model as a linear robust optimization problem considering the classic cardinality-restricted uncertainty set:

$$
\begin{aligned}
\operatorname{Maximize} & \left(\sum_{i \in N} \bar{r}_{i} x_{i}-\tau \Gamma-\sum_{i \in N} p_{i}\right) \\
\text { s.t. } & \sum_{i \in N} x_{i}=1, \\
& \tau+p_{i} \geq \hat{r}_{i} x_{i}, \quad \forall i \in N \\
& \tau, p_{i} \geq 0, \quad \forall i \in N \\
& x_{i} \in[0,1], \quad \forall i \in N,
\end{aligned}
$$

where $C$ is the total capital, $y_{i}$ is the portion of the investment in asset $i\left(x_{i}=y_{i} / C\right)$. The nominal value and the investment risk of return of asset $i$ are $\overline{r_{i}}$ and $\hat{r}_{i}$, respectively. The dual variables are $\tau$ and $p_{i}$, and $N$ the set of all assets. A less conservative approach (to avoid over-protection) is to compensate the robustness cost (the risk of obtaining lower profit) of "risky" assets, $\Psi$, by purchasing "safe" assets, $\Psi^{\prime}$. The safe assets can be either a bank savings account or a government bond [7]. Hence, the 
return value of a subset of assets, $\Psi^{\prime}$, are prone to be perturbed within the predefined uncertainty interval (note that $\overline{r_{i}}>\overline{r_{j}}$ and $\hat{r}_{i}>\hat{r}_{j}, \forall i \in \Psi, \forall j \in \Psi^{\prime}$ ). Given $\Phi$ as the budget of uncertainty,

$$
\Phi=\alpha \sum_{i \in \Psi^{\prime}} x_{i}^{*}
$$

where $\alpha$ is the robustness importance, the uncertainty set is defined as follows:

$$
U^{\Phi}=\left\{\tilde{r} \in R^{|\Psi|}: \tilde{r}_{i}=\bar{r}_{i} \pm \delta_{i} \hat{r}_{i}, \delta_{i} \in[0,1], \sum_{i \in \Psi} \delta_{i} \leq \Phi\right\} .
$$

Again, note that risk of asset $i$ is an internal property of return of asset $i$ (attribute-driven uncertainty); hence, we defined $\delta_{i}$ over a continuous domain. The budget of uncertainty varies depending on the combination of safe assets' pool. According to the worst-case approach, the problem is formally stated as follows:

$$
\begin{aligned}
\operatorname{Maximize} & \left(\sum_{i \in N} \bar{r}_{i} x_{i}-\max _{U^{\Phi}}\left\{\sum_{j \in \Psi} \hat{r}_{j} x_{j}^{*}\right\}\right) \\
\text { s.t. } & \sum_{i \in N} x_{i} \leq 1 \\
& x_{i} \in[0,1], \quad i \in N .
\end{aligned}
$$

The inner maximization function of problem (22) can be translated into a single max-LP problem (23) as follows:

$$
\begin{aligned}
\operatorname{Maximize} & \sum_{i \in \Psi} \hat{r}_{i} \delta_{i} x_{i}^{*} \\
\text { s.t. } & \sum_{i \in \Psi} \delta_{i} \leq \alpha \sum_{j \in \Psi^{\prime}} x_{j}^{*} \\
& \delta_{i} \in[0,1], \quad \forall i \in \Psi .
\end{aligned}
$$

Using dual of problem (23) and strong duality theorem, the robust counterpart is obtained as:

$$
\begin{aligned}
\text { Maximize } & \left(\sum_{i \in N} \bar{r}_{i} x_{i}-\tau\left(\alpha \sum_{i \in \Psi^{\prime}} x_{i}\right)-\sum_{i \in \Psi} p_{i}\right) \\
\text { s.t. } & \sum_{i \in N} x_{i} \leq 1 \\
& p_{i}+\tau \geq \hat{r}_{i} x_{i}, \quad \forall i \in \Psi \\
& \tau, p_{i} \geq 0, \quad \forall i \in \Psi \\
& x_{i} \in[0,1], \quad \forall i \in N
\end{aligned}
$$


Proposition 3.1. $\tau \leq \max _{i \in \Psi}\left(\hat{r}_{i}\right)$.

Proof. Consider deciding to invest the total capital on risky asset $k \in \Psi$ with the highest return, $\overline{r_{k}}=$ $\max _{i \in \Psi}\left(\overline{r_{i}}\right)$, and also the highest risk, $\hat{r}_{k}=\max _{i \in \Psi}\left(\hat{r}_{i}\right)$. Hence, $x_{k}=1$ and $p_{k}+\tau \geq \hat{r_{k}}$. According to the objective function, variables $p_{k}$ and $\tau$ should take the minimum possible values. Variable $p_{k}$ does not appear in any other constraint and takes value zero; therefore, the minimum possible value for $\tau$ is $\hat{r}_{k}$. In other words, variable $\tau$ cannot exceed $\max _{i \in \Psi}\left(\hat{r}_{i}\right)$.

The objective of problem (24) is not linear due to the quadratic term $\tau\left(\alpha \Sigma_{i \in \Psi^{\prime}} \mathrm{X}_{\mathrm{i}}\right)$. We reformulate this model based on the McCormick convex envelope relaxation [8] and the following properties:

$$
\begin{gathered}
\lambda_{i}=\tau y_{i}, \quad \forall i \in \Psi^{\prime} \\
0 \leq x_{i} \leq 1, \quad \forall i \in \Psi^{\prime} \\
0 \leq \tau \leq \max _{i \in \Psi}\left(\hat{r}_{i}\right) .
\end{gathered}
$$

Note that the efficiency of the relaxation heavily depends on the tightness of the bounds of the quadratic variables. The linear model of the robust counterpart is presented as follows:

$$
\begin{aligned}
\text { Maximize } & \left(\sum_{i \in N} r_{i} x_{i}-\alpha \sum_{i \in \Psi^{\prime}} \lambda_{i}-\sum_{i \in \Psi} p_{i}\right) \\
\text { s.t. } & \sum_{i \in N} x_{i} \leq 1, \\
& p_{i}+\tau \geq \hat{r}_{i} x_{i}, \quad \forall i \in \Psi, \\
& \lambda_{i} \leq \max _{j \in \Psi}\left(\hat{r}_{j}\right) x_{i}+\tau-\max _{j \in \Psi}\left(\hat{r}_{j}\right), \quad \forall i \in \Psi^{\prime}, \\
& \lambda_{i} \leq \max _{j \in \Psi}\left(\hat{r}_{j}\right), \quad \forall i \in \Psi^{\prime}, \\
& \lambda_{i} \leq \tau, \quad \forall i \in \Psi^{\prime}, \\
& \tau \leq \max _{j \in \Psi}\left(\hat{r}_{j}\right), \\
& \lambda_{i}=0, \quad \forall i \in \Psi \\
& \tau, p_{i} \geq 0, \quad \forall i \in \Psi \\
& \lambda_{i} \geq 0, \quad \forall i \in \Psi^{\prime} \\
& x_{i} \in[0,1], \quad \forall i \in N .
\end{aligned}
$$

\section{Experimental Analysis}

In the previous section, we developed different uncertainty sets for constructing robust counterpart optimization formulations; however, a natural question is: how efficient are the proposed uncertainty sets as compared to the cardinality-restricted uncertainty sets? The decisionmaker essentially wants to adopt the least conservative model (the best possible objective value) while the same probability of constraint violation is satisfied. 
Consider a binary knapsack problem with uncertain parameters in $\tilde{w}$. It is assumed that the weights $\tilde{w}$ are uncertain; identically, independently, and uniformly distributed in $\left[\bar{w}_{i}, \bar{w}_{i}+\hat{w}_{i}\right]$. Then, the robust counterpart is modelled as follows:

$$
\begin{aligned}
\operatorname{Maximize} & \sum_{i \in N} c_{i} x_{i} \\
\text { s.t. } & \sum_{i \in N} \bar{w}_{i} x_{i}+\Delta+\sum_{i} p_{i} \leq b \\
& p_{i}+\tau \geq \hat{w}_{i} x_{i}, \quad \forall i \in N \\
& \Delta, \tau, p_{i} \geq 0, \quad \forall i \in N \\
& x_{i} \in\{0,1\}, \quad \forall i \in N,
\end{aligned}
$$

where $\Delta$ for ordinary cardinality-restricted set $\left(U^{\Gamma}\right)$ is the product of dual variable $\tau$ and budget of uncertainty $\Gamma$, that is,

$$
\Delta=\tau \Gamma
$$

and for the variable budgeted set $\left(U^{\Phi}\right)$ is:

$$
\Delta=\left\{\sum_{i \in \Pi} \lambda_{i} \mid \lambda_{i} \geq-\max _{i \in N}\left(\hat{w}_{i}\right)\left(1-x_{i}\right)+\tau, \quad \forall i \in \Pi\right\} .
$$

The first experiment is conducted based on problem (29) using IBM Ilog CPLEX 12.6 with $|N|=100$. The capacity limit, $b$, is set to 1000 . The nominal weights, $\bar{w}_{i}$, and the costs, $c_{i}$, are randomly chosen from the sets $\{21,22, \ldots, 29\}$ and $\{16,17, \ldots, 77\}$, respectively. The weight uncertainty $\hat{w}_{i}$ is set to 10 percent of the nominal weight. Items of the set $\Pi$ is randomly chosen from set $N \cup\{0\}$ and uniformly distributed in $[0, \Gamma]$. Each simulation run is replicated 100 times for each solution and the mean of the objective values is reported.

The aim of this experiment is to analyze the performance of the proposed uncertainty sets in different protection levels. We refer to a measure, the price of robustness, originally proposed by Bertsimas and Sim [4], as a tradeoff between the level of protection against the value of the objective function under uncertainty. Suppose that $\xi_{D}$ and $\xi_{R}$ are the objective values of the robust and deterministic models, respectively. On the other hand, an $\alpha$-protected solution of a problem is defined as the solution with the probability of violation less than (1- $\alpha) \%$. Hence, the price of robustness under $\alpha$-protected solution $\left(\mathrm{PoR}^{\alpha}\right)$ for a maximization problem can be determined as follows.

$$
\operatorname{PoR}^{\alpha}=\frac{\xi_{D}-\xi_{R}^{\alpha}}{\xi_{D}}
$$

The less $\operatorname{PoR}^{\alpha}$ is, the better objective value is at hand. The probability of violation is defined as follows: 


$$
\operatorname{Pr}\left\{\sum_{i \in N} \bar{w}_{i} x_{i}^{*}+\Delta>b\right\} \leq 1-\alpha,
$$

where $x^{*}$ is the solution to the robust counterpart. The $\mathrm{PoR}^{\alpha}$ of the robust models based on two uncertainty sets is compared under three different probabilities of violation as illustrated in Table 1. Under zero protection level, $\Gamma=0(|\Pi|=0)$, obviously, $\operatorname{PoR}^{\alpha}$ is zero, since $\xi_{R}$ exactly equals $\xi_{D}$. Table 1 shows that, except for the zero and full protection level, $\operatorname{PoR}^{\alpha}$ of $U^{\Gamma}$ is strictly greater than that of $U^{\Phi}$. It is evident that different $\operatorname{PoR}^{\alpha}$ of $U^{\Gamma}$ observes the trade-off between the worst-case outcome and the probability of violation. The blank places in Table 1 indicate that solutions could not be found with the probability of violation less than (1- $\alpha$ ) for the robust counterpart protected with $U^{\Gamma}$. However, the variable budgeted set adjusts the solutions to obtain better $\operatorname{PoR}^{\alpha}$ with the probability of violation less than (1- $\alpha$ ). In other words, it automatically decreases the intensity of the worst-case outcome to meet the imposed probability of violation. Empirically, the following result can be inferred from Table 1:

$$
\operatorname{Pr}\left\{\sum_{i \in N} \bar{w}_{i} x_{i}^{*}+\Delta>b\right\}^{U^{\Gamma}} \leq \operatorname{Pr}\left\{\sum_{i \in N} \bar{w}_{i} x_{i}^{*}+\Delta>b\right\}^{U^{\Phi}} \leq 1-\alpha .
$$

\begin{tabular}{|c|c|c|c|c|c|c|}
\hline \multirow[b]{2}{*}{$\Gamma(|\Pi|)$} & \multicolumn{2}{|c|}{$1-\alpha=0.1$} & \multicolumn{2}{|c|}{$1-\alpha=0.05$} & \multicolumn{2}{|c|}{$1-\alpha=0.025$} \\
\hline & $U^{\Gamma}$ & $U^{\Phi}$ & $U^{\Gamma}$ & $U^{\Phi}$ & $U^{\Gamma}$ & $U^{\Phi}$ \\
\hline 0 & 0.0000 & 0.0000 & 0.0000 & 0.0000 & 0.0000 & 0.0000 \\
\hline 10 & 0.0192 & 0.0049 & 0.0232 & 0.0079 & 0.0205 & 0.0100 \\
\hline 20 & 0.0353 & 0.0199 & 0.0439 & 0.0150 & 0.0386 & 0.0197 \\
\hline 30 & 0.0497 & 0.0241 & 0.0628 & 0.0300 & 0.0544 & 0.0316 \\
\hline 40 & 0.0616 & 0.0353 & 0.0767 & 0.0407 & - & 0.0359 \\
\hline 50 & 0.0668 & 0.0395 & 0.0767 & 0.0414 & - & 0.0324 \\
\hline 60 & 0.0668 & 0.0448 & 0.0767 & 0.0493 & - & 0.0371 \\
\hline 70 & 0.0668 & 0.0514 & 0.0767 & 0.0550 & - & 0.0729 \\
\hline 80 & 0.0668 & 0.0532 & 0.0767 & 0.0639 & - & 0.2107 \\
\hline 90 & 0.0668 & 0.0626 & 0.0767 & 0.0728 & - & - \\
\hline 100 & 0.0668 & 0.0668 & 0.0767 & 0.0767 & - & - \\
\hline
\end{tabular}

Table 1. Comparing the price of robustness under $\alpha$-protected solutions

The second experiment examines $\operatorname{PoR}^{\alpha}$ in different problem sizes. The details are presented in Table 2. It is assumed that the weight and utility parameters are uniformly distributed in $\left[w_{\min }, w_{\max }\right]$ and $\left[c_{\min }, c_{\max }\right]$, respectively. Figure 1 illustrates the resulting $\operatorname{PoR}^{\alpha}$ values for the robust counterparts protected with $U^{\Gamma}$ and $U^{\Phi}$ in a moderate violation percentage $(\alpha=0.05)$. The value of $\Gamma(|\Pi|)$ is chosen according to the fact that further increase in $\mathrm{PoR}^{\alpha}$ is not possible. For example, according to Table 1 and $1-\alpha=0.1$, this value lies between 40 and 50. Each problem is replicated with the 100 different random parameter configurations and the mean of $\mathrm{PoR}^{\alpha}$ is considered. Again, the less $\mathrm{PoR}^{\alpha}$ means the better performance of uncertainty set and Figure 1 sheds light on the merit of $U^{\Phi}$. 
Table 2. Parameters configurations for different instances

\begin{tabular}{|c|c|c|c|c|c|}
\hline Problem No. & 1 & 2 & 3 & 4 & 5 \\
\hline$(\mathrm{b},|\mathrm{N}|)$ & $(100,10)$ & $(200,20)$ & $(300,30)$ & $(400,40)$ & $(500,50)$ \\
\hline $\mathrm{C}[\mathrm{cmin}, \mathrm{cmax}]$ & {$[16,30]$} & {$[18,35]$} & {$[20,40]$} & {$[22,45]$} & {$[22,50]$} \\
\hline $\mathrm{W}$ [wmin,wmax] & {$[15,20]$} & {$[16,21]$} & {$[18,23]$} & {$[20,27]$} & {$[23,33]$} \\
\hline Problem No. & 6 & 7 & 8 & 9 & 10 \\
\hline$(\mathrm{b},|\mathrm{N}|)$ & $(600,60)$ & $(700,70)$ & $(800,70)$ & $(800,80)$ & $(1000,100)$ \\
\hline $\mathrm{C}[\mathrm{cmin}, \mathrm{cmax}]$ & {$[24,55]$} & {$[24,60]$} & {$[26,65]$} & {$[28,70]$} & {$[28,75]$} \\
\hline $\mathrm{W}$ [wmin,wmax] & {$[28,38]$} & {$[29,40]$} & {$[31,46]$} & {$[31,48]$} & {$[31,50]$} \\
\hline
\end{tabular}

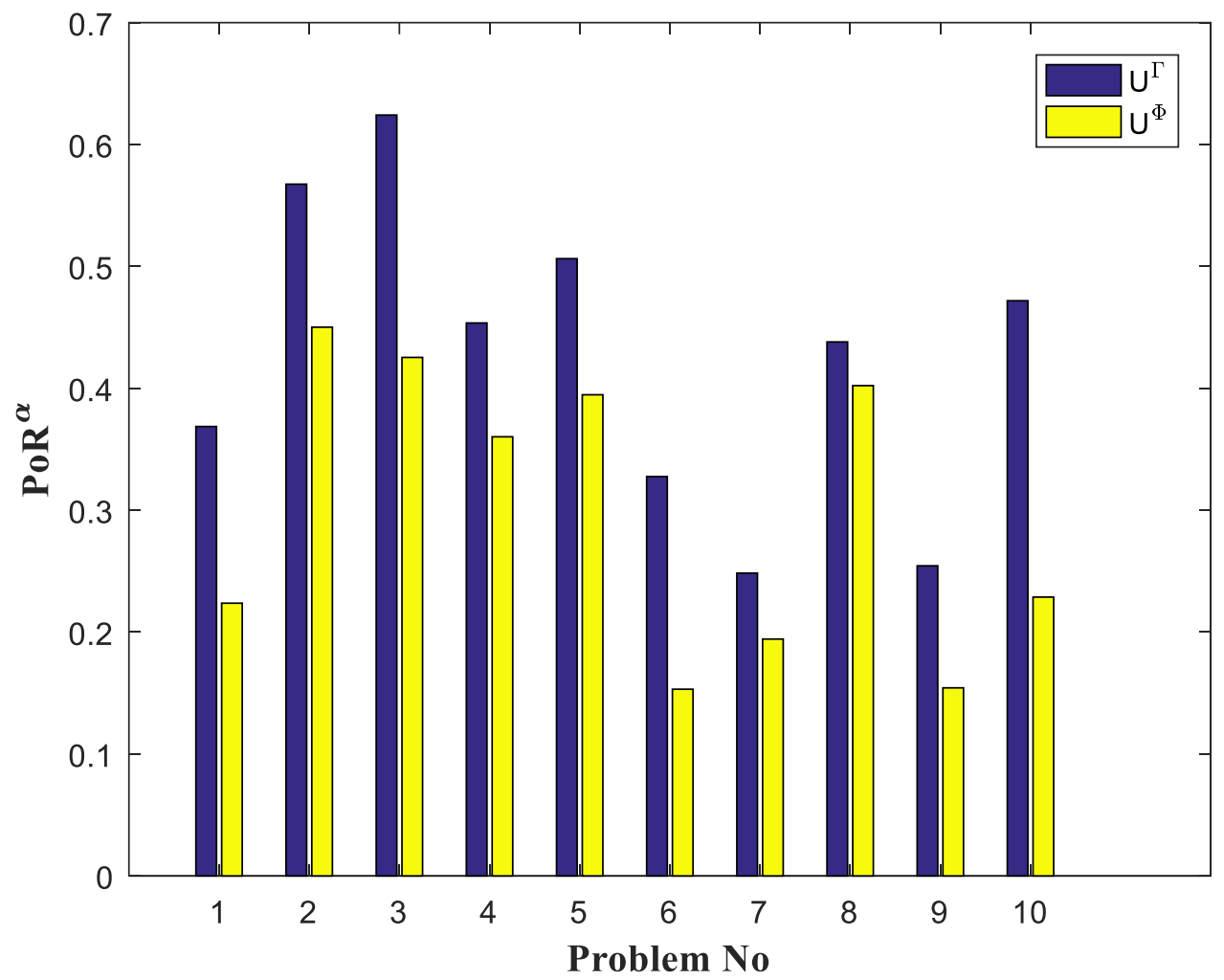

Figure 1. Comparing the price of robustness for different problem sizes

\section{Conclusion}

We investigated structural properties of uncertain events to obtain intelligent robust models with refined uncertainty polytopes. We also illustrated the approach by applying them to the two classic robust optimization problems. There are cases when polyhedral theorems and affine policies do not operate for the conversion of event-driven approaches to attribute-driven ones; hence, we recommend the adjustable robust models and we suggest it as a future direction of research. 


\section{Acknowledgments}

We would like to express our deepest appreciation to Willem-Jan Van Hoeve (Tepper School of Business at Carnegie Mellon University), Andre A. Cire (University of Toronto), and Noushin Mohseni (Tabriz University) who provided us the possibility to complete this study.

\section{References}

[1] An, Y., Zeng, B., Zhang, Y. and Zhao, L. (2014), Reliable p-median facility location problem: two-stage robust models and algorithms, Transportation Research Part B: Methodological, 64, 54-72.

[2] Ben-Tal, A. and Nemirovski, A. (1998), Robust convex optimization, Mathematics of Operations Research, 23(4), 769-805.

[3] Ben-Tal, A., Nemirovski, A. and Roos, C. (2002), Robust solutions of uncertain quadratic and conic-quadratic problems. SIAM Journal on Optimization, 13(2), 535-560.

[4] Bertsimas, D. and Sim, M. (2004), The Price of robustness, Operations Research, 52(1), $35-53$.

[5] El Ghaoui, L., Oustry, F. and Lebret, H. (1998), Robust solutions to uncertain semidefinite programs, SIAM Journal on Optimization, 9(1), 33-52.

[6] Falk, J.E. (1976), Technical Note-Exact Solutions of Inexact Linear Programs, Operations Research, 24(4), 783-787.

[7] Mankiw, N.G. (2014), Principles of Economics, Cengage Learning.

[8] Mitsos, A., Chachuat, B. and Barton, P.I. (2009), McCormick-based relaxations of algorithms, SIAM Journal on Optimization, 20(2), 573-601.

[9] Poss, M. (2013), Robust combinatorial optimization with variable budgeted uncertainty, 4OR, 11(1), 75-92.

[10] Soyster, A.L. (1973), Technical note - convex programming with set-inclusive constraints and applications to inexact linear programming, Operations Research, 21(5), 1154-1157.

[11] Wolsey, L.A. and George, L.N. (1988), Integer and Combinatorial Optimization (1st ed.), Wiley-Interscience.

[12] Zeng, B. (2013). Solving two-stage robust optimization problems using a column-andconstraint generation method, Operations Research Letters, 41(5), 457-461. 


\section{Appendix: Proof of Property 3.1}

This is equivalent to proving that the technological coefficient of $\Xi$ in the LP-relaxed form is totally unimodular (TU). Now, we make use of the following proposition proved in [11]. Let $\boldsymbol{Q}$ be a matrix in $\{0,1,-1\}$ with no more than two nonzero elements in each column. Then, $\boldsymbol{Q}$ is TU if and only if the rows of $Q$ can be partitioned into two subsets $\boldsymbol{Q}_{\mathbf{1}}$ and $\boldsymbol{Q}_{2}$ such that if a column contains two nonzero elements and both nonzero elements have the same sign, then one is in a row contained in $Q_{1}$ and the other is in a row contained in $\boldsymbol{Q}_{2}$.

In the LP-relaxed form, the constraint set $\delta_{i} \leq 1$ should also be added to $\Xi$. The technological coefficients of $\Xi$ are characterized as a matrix $\boldsymbol{A}^{\Xi}$ as follows:

$$
\boldsymbol{A}_{(|N|+1) \times|N|}^{\Xi}=\left(\begin{array}{c}
\boldsymbol{e}_{1 \times|N|} \\
\boldsymbol{I}_{|N| \times|N|}
\end{array}\right),
$$

where $\boldsymbol{e}$ is an all-ones vector and $\boldsymbol{I}$ is the identity matrix. $\boldsymbol{Q}=\boldsymbol{A}^{\boldsymbol{\Xi}}$ has components of 0 and +1 with no more than two nonzero entries in each column. $\boldsymbol{A}^{\boldsymbol{\Sigma}}$ can be partitioned into two subsets $\boldsymbol{Q}_{\mathbf{1}}$ and $\boldsymbol{Q}_{2}$. Consider $\boldsymbol{Q}_{\mathbf{1}}=\boldsymbol{e}$ and $\boldsymbol{Q}_{2}=\boldsymbol{I}$ (one of +1 is in a row contained in $\boldsymbol{Q}_{\mathbf{1}}$ and the other is in aw row contained in $\boldsymbol{Q}_{2}$ ). Under this conditions, according to the proposition proved in [11], $\boldsymbol{A}^{\boldsymbol{\Xi}}$ is TU. Thus, the relaxed $\Xi$ is integral for $\Gamma \in \mathbb{Z}$. 